\title{
Drug Induced Steven- Johnson Syndrome (SJS)
}

\author{
Riyaz Miya*, Ashok Kumar Malpani, Sangamesh Keri, Rohit Panagaon
}

Department of Pharmacy Practice, RMES College of Pharmacy, Kalaburagi, Karnataka, INDIA.

\begin{abstract}
Steven Johnson Syndrome (SJS) or Toxic Epidermal Necrolysis (TEN) is a life threatening immune complex mediated hypersensitivity reaction which mainly involves skin and mucous membrane. SJS is generally induced by the drugs. The manifestations include blisters on skin, facial swelling and hyper pigmentation. Here is a 28-yearold Indian male received Ofloxacin, Paracetamol and Phenobarbitone and experienced a severe skin reaction which was diagnosed as Stevens Johnson syndrome (SJS). The above drugs will be implicated in cases of Stevens Johnson Syndrome (SJS) / Toxic Epidermal Necrosis (TEN). There are few case reports of that have been associated with Stevens Johnson Syndrome (SJS)/ Toxic Epidermal Necrosis (TEN). We hope that this case report creates awareness to the health care professionals.
\end{abstract}

Key words: SJS, TEN, Ofloxacin, Paracetamol, Phenobarbitone.

\section{INTRODUCTION}

Adverse Drug Reactions (ADR) accounts $6 \%$ of the total hospital admission, increases economic burden on healthcare system, results into withdrawal of drugs from market and death. Among various ADR, cutaneous drug reactions mainly Stevens Johnson Syndrome (SJS) and Toxic Epidermal Necrolysis (TEN) are rare but potentially fatal reactions that endangers patient's life. ${ }^{1}$

Cutaneous drug reactions are the most common type of adverse drug reactions. The percentage of potentially serious reactions is around 2\%. Stevens Johnson Syndrome (SJS) and Toxic Epidermal Necrolysis (TEN) are potentially serious cutaneous reactions, characterized by high fever, wide-spread blistering exanthema of macules and atypical target-like lesions, accompanied by mucosal involvement. $^{2}$

Steven Johnson Syndrome is a rare autoimmune disorder which mainly includes skin and mucous membrane. It was reported that the incidence of SJS is 0.05 to 2 persons million per population per year. The reported mortality ranges from 3-10\%. The most common drugs which cause
SJS are sulfonamides, non-steroidal antiinflammatory drugs, imidazole antifungal, cephalosporins, anticonvulsants, allopurinol, broad-spectrum bactericidal agents and HAART regimen. Rarely fluoroquinolones may cause serious cutaneous drug reactions. Ofloxacin, an quinolone antimicrobials which belongs to first generation was first introduced in 1980. Oflaxacin inhibits the enzyme bacterial DNA-gyrase, which nicks double stranded DNA, introduce negative supercoils and then reseals the nicked ends. This is necessary to prevent excessive positive supercoiling of the strands when they separate to permit replication or transcription. The DNA-gyrase consists of two- A and two- B subunits. A subunits carries out nicking of DNA, B subunits introduces negative supercoils and then $A$ subunit reseals the strands. Ofloxacin binds to a subunit with high affinity and interfere with its strand cutting and resealing function. The common side effects likely to be found are dizziness, headache, restlessness, bad taste, anorexia etc. ${ }^{3}$

In a large, international, case-control study, the multivariate relative risk of toxic
DOI: 10.5530/ijopp.12.2.28

Address for correspondence: Riyaz Miya,

Assistant Professor, Department of Pharmacy Practice, RMES College of Pharmacy, Balaji Nagar, Old Jewaragi Road, Kalaburagi- 585102, Karnataka, INDIA.

Phone no: +91-9742498030

Email: patelriyaz84@gmail.com

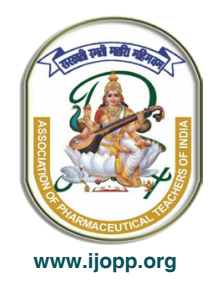


epidermal necrolysis or Stevens Johnson Syndrome was found to be 10 (95\% CI 2.6 to 38) for quinolone antibiotics. In other words, subjects exposed to quinolone antibiotics were 10 times more likely to experience either Stevens Johnson Syndrome or Toxic Epidermal Necrolysis than did subjects not exposed to quinolones. Three of the quinolone cases noted ofloxacin as the offending antibiotic. Details of the cases were not given, so it is not known whether these reactions were Stevens Johnson Syndrome or Toxic Epidermal Necrolysis. However, a MEDLINE search from 1966 to December 2000 revealed no reports of toxic epidermal necrolysis, erythema multiform or Stevens Johnson Syndrome due to ofloxacin therapy. This is a report of a fatal case of Toxic Epidermal Necrolysis associated with the use of oral ofloxacin. ${ }^{4}$

\section{Case Description}

A 28-year-old male patient who was admitted to Dermatology ward at Gulbarga Institute of Medical Sciences, Kalburagi on 14/11/2018 with chief complaints of skin rashes over the body with burning eyes and difficulty in swallowing (Figure 1). On examination hyper pigmented skin, minute blisters initially around both the eyes and lips, then all over the body. The patient had abnormal birth and mental illness (after birth first five days didn't feed) and he is a known case of Seizures since 20 years and on and off medication Tablet Phenobarbitone P.O 60mg OD.

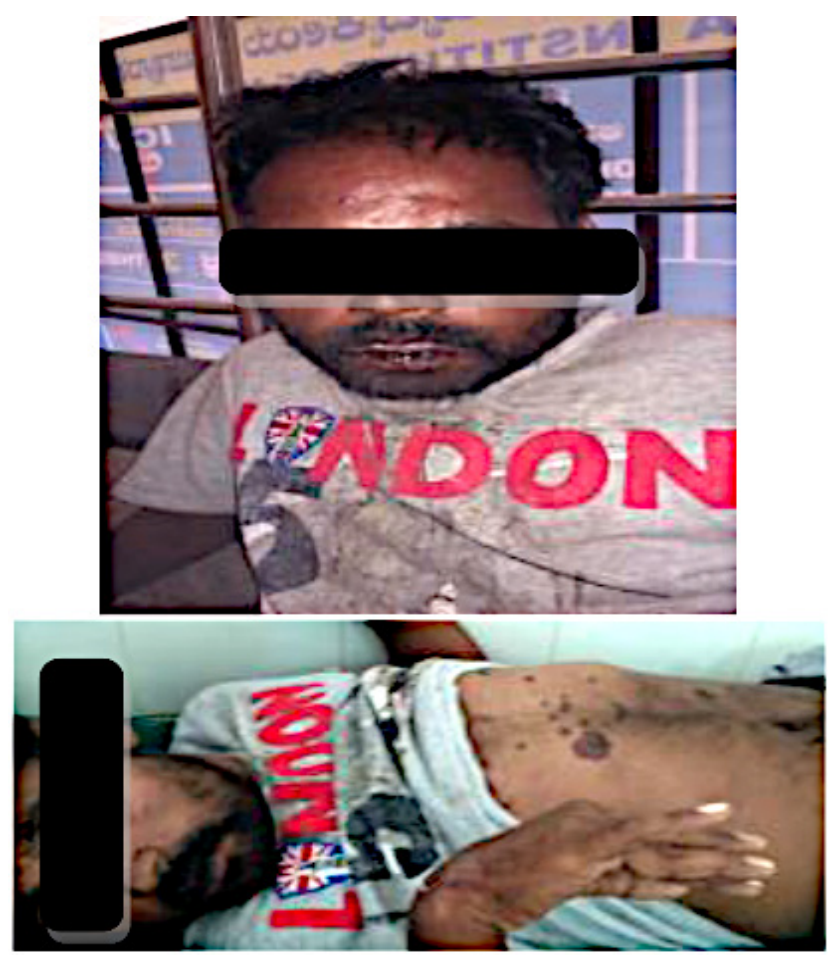

Figure 1: Hyper pigmented skin, minute blisters around both the eyes, lips and all over the body.
His Father revealed the present medication history of Tablet Ofloxacin P.O 200mg BD, Tablet Paracetamol P.O 500mg TID and Capsule Omeprazole + Domperidone P.O (20mg+10mg) OD for the treatment of fever and chills at local Doctor. After taking the prescribed medications patient had developed fever and swelling over the face. Then patient has been taken to another physician at private clinic and he has been prescribed with Tablet Paracetamol P.O 500mg TID, Tablet Domperidone PO $10 \mathrm{mg}$ OD and syrup Salbutamol sulphate P.O $10 \mathrm{ml}$ BD. After taking this medication patient had no relief from symptoms. Then patient consulted to the Department of Dermatology at Gulbarga Institute of Medical Sciences, Kalaburagi, where on clinical examination he has been diagnosed as Drug Induced Steven Johnson Syndrome (SJS) and treatment has been given for the same. The patient's recovery achieved in 8 days.

\section{DISCUSSION}

As per our observation it is drug induced SJS. As patient had taken multiple drugs, like Paracetamol P.O 500mg TID, Ofloxacin P.O 200mg and Phenobarbitone P.O $60 \mathrm{mg}$ OD in several studies reveals that these all three drugs will cause SJS. Phenobarbitone and Paracetamol have a moderate interaction that can more prone to SJS. This patient did not appear to have any reason for developing toxic epidermal necrolysis other than the addition of Ofloxacin and Paracetamol to his treatment regimen. He had not received any recent immunizations. He did not have a viral infection, graft-versus-host disease, hematologic malignancy, systemic lupus erythematosus, known cerebral tumor and upper respiratory infection.

The study carried out by Stephen L Melde revealed that, there is very little published information regarding ofloxacin-induced toxic epidermal necrolysis. There are few cases reports with other fluoroquinolones in which they have been associated with toxic epidermal necrolysis. It ishoped that this case report creates awareness that ofloxacin induced toxic epidermal necrolysis is possible.

The study conducted by Neki et al. concluded that Acetaminophen is a relatively safe drug devoid of serious side effects, catastrophic severe hypersensitivity reactions can occur with its usage, which can be potentially life threatening. This case highlights the fact that no drug is completely safe and the treating physician should always be careful even while handling "potentially safe" drugs. Although litigation suits are not a routine in India, things are changing thick and fast. As on one hand; proper guidance and explanation of illness, treatment, procedures and potential complications to all patients

Indian Journal of Pharmacy Practice, Vol 12, Issue 2, Apr-Jun, 2019 
is beneficial for their own interests; it saves the doctors from unnecessary troubles as well. ${ }^{5}$

The study carried out by Purkayastha et al. revealed that Paracetamol is capable of causing such reactions as is evident from earlier reports. It is thus very essential for the clinicians to be aware and alert of severe undesirable hypersensitivity reactions that might occur even with drugs that are thought to be possibly safe such as Paracetamol. $^{6}$

The study carried out by Lihite et al. revealed that, the antimicrobials, anticonvulsants and antipyretics were commonly reported offending group of drugs for SJS/ TEN in patients. ${ }^{1}$ Paracetamol $(8 / 45)$ and Phenytoin (7/45) were commonly reported offending drugs in individual drug category. Most of the cases were categorized very probable as per ALDEN whereas by WHO*_UMC causality assessment system most of the cases were probable. Since the number of cases studied is less, it warrants further research.

The study carried out by Naveen et al. observed that, Oflaxacin, a commonly used antibiotic in India, has a risk of inducing SJS TEN, which may be fatal. SJS-TEN induced by ofloxacin has a higher morbidity and mortality compared to anti convulsant. ${ }^{2}$ Anti-epileptics also have a potential for causing this serious adverse reaction. Since the number of cases studied is less, it warrants further research.

\section{CONCLUSION}

In our study we have identified that patient had developed SJS after taking Ofloxacin, Paracetamol and Phenobarbitone. It is difficult to confirm which drug has induced the SJS. As our patient has a history of epilepsy since 20 years, he is more prone to develop SJS / TEN. Since the number of case studies are less so we opine still further research to confirm the same.

\section{ACKNOWLEDGEMENT}

Authors greatly appreciate the assistance and Support given by the Health Care Staff of Gulbarga Institute of Medical Sciences, Kalaburagi.

\section{CONFLICT OF INTEREST}

Authors declare no conflict of interest.

\section{ABBREVIATIONS}

ADR: Adverse drug reactions; SJS: Stevens Johnson syndrome; TEN: Toxic Epidermal Necrolysis; HAART: Highly active anti-retro therapy; CI: Class interval; Mg: Mile gram; P.O: By Mouth; OD: Once in day; BD: Bis in day; TID: Thrice in day; ML: Mile liter.

\section{SUMMARY}

Our study aims to minimize the ADR like SJS by making alert to physicians and other healthcare professionals intern we can avoid hospital admissions because of ADR, reduce economic burden of the patients and health related quality of life of the patient can be improved.

\section{REFERENCES}

1. Lihite RJ, et al. A study on drug induced SJS, TEN and SJS-TEN overlap. J Young Pharm. 2016;8(2):149-53.

2. Naveen $\mathrm{KN}$, et al. An analysis of Stevens Johnson Syndrome. Indian Journal of Pharmacology. 2013;45(1):80-2.

3. James VJ, et al. Ofloxacin induced Steven Johnson Syndrome- case report. Asian Pac J Health Sci. 2015;2(2):10-1.

4. Stephen LM. Ofloxacin: a Probable Cause of Toxic Epidermal Necrolysis. The Annals of Pharmacotherapy. 2001;35(1):1398-40.

5. Neki, et al. Paracetamol induced Steven-Johnson syndrome-toxic epidermal necrolysis overlap. International Journal of Medical and Health Research. 2016;2(12):59-61.

6. Purkayastha A, et al. A Case Report of Paracetamol-Induced Toxic Epidermal Necrolysis in A Female Patient at A Tertiary Care Hospital in Assam. Asian J Pharm Clin Res. 2016;9(4):7-8. 